\title{
Editorial
}

\section{Chronic Coronary Total Occlusion}

Chronic total occlusion (CTO) is quite frequent finding in coronary artery disease. Comprised of a combination of fibrocalcific and thrombotic elements, CTO has been reported in approximately onethird of patients undergoing diagnostic coronary angiography. This thematic issue goes in depth into "coronary CTO" that still represents the last frontier in percutaneous coronary intervention (PCI). Historically, CTO remains the most challenging lesion subset for PCI. Despite a growing body of evidence pertaining to favourable effects of revascularization, interventional cardiologists are quite reluctant to attempt CTO PCI which might be attributed to historically low success rate, increased cost, prolonged procedure time and the lack of well defined approach.

This expertly organized and carefully written issue represents the collective view of CTO PCI ex-

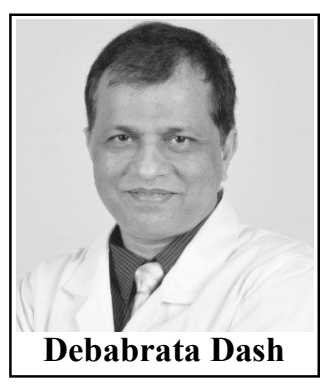
perts across the world. Many of the historical barriers have been removed as would be revealed in the articles. Combinations of several innovative techniques and technologies are tested for enhancement of procedural success allowing the operators a greater opportunity for safe and effective PCI.

This issue starts with comprehensive description of applied pathology for CTO intervention followed by importance of collateral circulation with an interventional perspectives. Emergence of advances in numerous techniques and technologies has refined the strategies of antegrade and retrograde approaches leading to remarkable improvements in procedural success and complication rate. The hybrid approach is intended to create a process, whereby, the operators react to anatomy and craft strategies accordingly. Over the course of this issue, the reader will be acquainted with complications of these procedures, steps towards preventing them, and radiation management. The imaging modalities like intravascular ultrasound and multislice computed tomography coronary angiography play pivotal role in CTO treatment. Finally, the general approach for training standards and recommendations for CTO PCI is outlined with respect to program and individual requirements. Surely, the assimilation of important practical materials amassed in this issue would go a long way to improve the technical skill of the operators and strive for the best procedural and clinical success. Vice chairperson of Asia Pacific Association of Interventional Cardiology (APAIC)

Honorary Professor, Beijing Tiantan Hospital, Capital University, Beijing Visiting Professor, Tan Tao University, Vietnam Formerly Fellow in interventional cardiology in VGH, Taipei Fellow in complex angioplasty in Toyohashi Heart center, Japan E-mail:dr_dash2003@yahoo.com 